\title{
Some Stable Difference Approximations to a Fourth-Order Parabolic Partial Differential Equation
}

\section{By G. Fairweather and A. R. Gourlay}

1. Introduction. Consider the fourth-order parabolic partial differential cquation

$$
\frac{\partial^{2} u}{\partial t^{2}}+\frac{\partial^{4} u}{\partial x^{4}}=0, \quad 0 \leqq x \leqq 1, t>0,
$$

subject to the initial conditions

$$
\begin{aligned}
u(x, 0) & =g_{0}(x) \\
\frac{\partial u}{\partial t}(x, 0) & =g_{1}(x)
\end{aligned}
$$

for $0 \leqq x \leqq 1$, and the boundary conditions

$$
\begin{array}{rlrl}
u(0, t) & =f_{0}(t), \quad & u(1, t)=f_{1}(t), \\
\frac{\partial^{2} u}{\partial x^{2}}(0, t) & =p_{0}(t), \quad \frac{\partial^{2} u}{\partial x^{2}}(1, t)=p_{1}(t), \quad t \geqq 0 .
\end{array}
$$

Following Richtmyer [11] we introduce two new variables $\Phi$ and $\Psi$ defined by

$$
\Phi=\frac{\partial u}{\partial t}, \quad \Psi=\frac{\partial^{2} u}{\partial x^{2}} .
$$

Eq. (1a) can now be rewritten as two simultaneous partial differential equations of the form

$$
\frac{\partial \Phi}{\partial t}=-\frac{\partial^{2} \Psi}{\partial x^{2}} ; \quad \frac{\partial \Psi}{\partial t}=\frac{\partial^{2} \Phi}{\partial x^{2}}
$$

or as the second-order system

$$
\frac{\partial \Omega}{\partial t}=A \frac{\partial^{2} \Omega}{\partial x^{2}}
$$

where

$$
\mathbf{\Omega}=\left[\begin{array}{l}
\Phi \\
\Psi
\end{array}\right] \text { and } A=\left[\begin{array}{cc}
0 & -1 \\
1 & 0
\end{array}\right] .
$$

Since $A+A^{\prime}=0$ and $A^{-1}=-A,(4)$ is a Schrödinger type system of partial differential equations, (Kreiss [6]).

Richtmyer [11] and Evans [5] have derived finite difference methods for the numerical solution of Eq. (4) which are based on well-known algorithms for the numerical solution of the scalar equation

$$
\frac{\partial v}{\partial t}=\frac{\partial^{2} v}{\partial x^{2}}
$$

Received February 21, 1966. 
In this paper, explicit and implicit finite difference methods based on the semiexplicit method of Lees [7] and the high accuracy method of Douglas [3] respectively are formulated for the numerical solution of Eq. (4), and algorithms for the determination of the solution $u$ of (1) from the calculated values of $\Phi$ and $\Psi$ derived. By means of numerical experiments, these methods will be compared with those of Richtmyer and Evans. In addition, the new explicit and implicit methods will be generalised to the case of variable coefficients. Finally, finite difference schemes for the solution of the equation

$$
\frac{\partial^{2} u}{\partial t^{2}}+\nabla^{4} u=0, \quad \text { where } \nabla^{2} \equiv \frac{\partial^{2}}{\partial x^{2}}+\frac{\partial^{2}}{\partial y^{2}},
$$

subject to appropriate initial and boundary conditions are considered.

2. Explicit Methods. A rectangular network of points with mesh sizes $h$ and $k$ in the $x$ and $t$ directions respectively, where $N h=1$ is superimposed on the region $0 \leqq x \leqq 1, t \geqq 0$. The values of the functions $\Phi(x, t), \Psi(x, t)$ and $\Omega(x, t)$ at the mesh points $x=i h, t=n k(i=0,1, \cdots, N ; n=0,1, \cdots)$ are given by $\phi_{i, n}$, $\psi_{i, n}$ and $\omega_{i, n}$ respectively.

The method of Evans [5] may be derived by applying the method of Dufort and Frankel [4] for the solution of (5) to Eq. (4) to obtain the finite difference formula

$$
\omega_{i, n+1}-\omega_{i, n-1}=2 r A\left[\omega_{i+1, n}+\omega_{i-1, n}-\omega_{i, n-1}-\omega_{i, n+1}\right]
$$

i.e.

$$
(I+2 r A) \omega_{i, n+1}=(I-2 r A) \omega_{i, n-1}+2 r A\left[\omega_{i+1, n}+\omega_{i-1, n}\right]
$$

where $r=k / h^{2}$ and $I$ is the $2 \times 2$ unit matrix. Since

$$
(I+2 r A)^{-1}=(I-2 r A)\left(1+4 r^{2}\right)
$$

and $A^{2}=-I$, Eq. (6) may be written in the explicit form

$$
\boldsymbol{\omega}_{i, n+1}=\left(a_{2 r} I-2 b_{2 r} A\right) \omega_{i, n-1}+\left(c_{2 r} I+b_{2 r} A\right)\left[\omega_{i+1, n}+\omega_{i-1, n}\right],
$$

where $a_{\alpha}=\left(1-\alpha^{2}\right) /\left(1+\alpha^{2}\right), b_{\alpha}=\alpha /\left(1+\alpha^{2}\right), c_{\alpha}=\alpha^{2} /\left(1+\alpha^{2}\right)$.

Writing (7) in terms of the components of $\omega$, we obtain Evans' formulation

$$
\begin{aligned}
& \phi_{i, n+1}=a_{2 r} \phi_{i, n-1}+c_{2 r}\left(\phi_{i+1, n}+\phi_{i-1, n}\right)-b_{2 r}\left(\psi_{i+1, n}+\psi_{i-1, n}-2 \psi_{i, n-1}\right) \\
& \psi_{i, n+1}=a_{2 r} \psi_{i, n-1}+c_{2 r}\left(\psi_{i+1, n}+\psi_{i-1, n}\right)+b_{2 r}\left(\phi_{i+1, n}+\phi_{i-1, n}-2 \phi_{i, n-1}\right) .
\end{aligned}
$$

This scheme is unconditionally stable, and has local truncation errors of

$$
O\left(h^{2}+k^{2}+(k / h)^{2}\right) \text {. }
$$

However, it is a three level scheme and so requires starting values on $t=k$ as well as on $t=0$.

A finite difference method for the solution of (4) which involves only two time levels is that based on the semiexplicit method of Lees [7]. This scheme may be written in the form

$$
\omega_{i, n+1}-\omega_{i, n}=r A\left[\omega_{i-1, n+1}+\omega_{i+1, n}-\omega_{i, n+1}-\omega_{i, n}\right]
$$


or

$$
(I+r A) \omega_{i, n+1}=(I-r A) \omega_{i, n}+r A\left[\omega_{i-1, n+1}+\omega_{i+1, n}\right]
$$

from which, on premultiplication by $(I+r A)^{-1}$, we obtain

$$
\omega_{i, n+1}=\left(a_{r} I-2 b_{r} A\right) \omega_{i, n}+\left(c_{r} I+b_{r} A\right)\left(\omega_{i+1, n}+\omega_{i-1, n+1}\right) .
$$

In terms of the components $\phi_{i, n}, \psi_{i, n}$ this scheme becomes

$$
\begin{aligned}
& \phi_{i, n+1}=a_{r} \phi_{i, n}+c_{r}\left(\phi_{i+1, n}+\phi_{i-1, n+1}\right)-b_{r}\left(\psi_{i+1, n}+\psi_{i-1, n+1}-2 \psi_{i, n}\right) \\
& \psi_{i, n+1}=a_{r} \psi_{i, n}+c_{r}\left(\psi_{i+1, n}+\psi_{i-1, n+1}\right)+b_{r}\left(\phi_{i+1, n}+\phi_{i-1, n+1}-2 \phi_{i, n}\right) .
\end{aligned}
$$

It should be noted that formulas (10a) and (10b) must be computed alternately, whereas the unknowns $\phi_{j, n+1}, \psi_{j, n+1}(j=1, \cdots, N-1)$ in (8) need only be calculated line by line. The local truncation errors of this method are

$$
O\left(h^{2}+k+(k / h)\right)
$$

and so the semiexplicit method appears theoretically to be less accurate than the method of Dufort and Frankel for the solution of (4). Both methods have the disadvantage that, if $k$ and $h$ tend to zero at the same rate, their respective solutions do not converge to the solution of (4), (see Evans [5], Lees [7]).

The stability of (9) is analysed by the method of Richtmyer [11]. We substitute

$$
\boldsymbol{\omega}_{m, n}=\varrho_{n} e^{i \beta x}, \text { where } \varrho_{n}=\left[\begin{array}{l}
\tilde{a}_{n} \\
\tilde{b}_{n}
\end{array}\right]
$$

and $x=m h,(m=1, \cdots, N-1)$, in (9) to obtain

$$
\varrho_{n+1}=G \varrho_{n}
$$

where $G$, the amplification matrix or symbol, is given by

$$
G=\frac{1}{1+l^{2}}\left[\begin{array}{cc}
1-|l|^{2} & -2 \operatorname{Re} l \\
2 \operatorname{Re} l & 1-|l|^{2}
\end{array}\right]
$$

where $l=r\left[1-e^{i \beta h}\right]=-2 i r e^{i \beta h / 2} \sin (\beta h / 2)$ and hence $\operatorname{Re} l=2 r \sin ^{2}(\beta h / 2)$. It follows that $G^{*} G=I$ and so the method is unconditionally stable, (Richtmyer [11]).

3. Implicit Methods. The most widely used implicit methods for the numerical solution of the scalar equation (5) are the Crank-Nicolson method [2] and the high order correct difference method (H.O.C.M.) of Douglas [3]. When the former method is applied to the vector equation (4), we obtain the algorithm used by Richtmyer in the form

$$
\left(I-\frac{1}{2} r A \delta_{x}^{2}\right) \omega_{i, n+1}=\left(I+\frac{1}{2} r A \delta_{x}^{2}\right) \omega_{i, n},
$$

where $\delta_{x}{ }^{2}$ is the usual central difference operator in the $x$ direction. The totality of difference equations of type (12) at each time step leads to a system of $(N-1)$ linear equations for the $(N-1)$ unknowns

$$
\omega_{i, n}=\left[\begin{array}{l}
\phi_{i, n} \\
\psi_{i, n}
\end{array}\right], \quad(i=1, \cdots, N-1),
$$


which may be written in the form

$$
Q \mathrm{~W}_{n+1}=Q \mathrm{~W}_{n}+\mathbf{k}
$$

where $\mathrm{W}_{m}=\left[\omega_{1, m}, \cdots, \omega_{N-1, m}\right]^{T}, m=n, n+1$, and $\mathbf{k}$ is a constant vector arising from the boundary conditions. The matrices $Q$ and $B$ are tridiagonal matrices of order $2(N-1)$ which may be written in the block forms

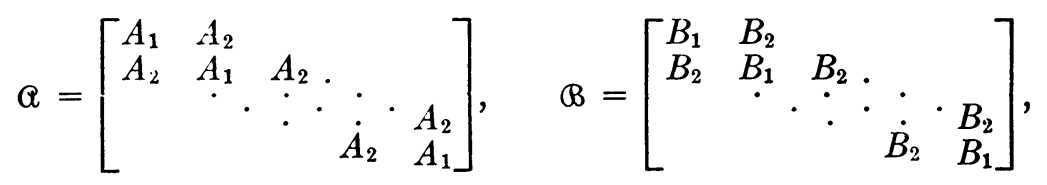

where the $2 \times 2$ matrices $A_{i}, B_{i},(i=1,2)$ are given by

$$
A_{1}=I+r A, \quad A_{2}=-\frac{1}{2} r A, \quad B_{1}=I-r A, \quad B_{2}=\frac{1}{2} r A .
$$

This system of equations may be solved by the well-known algorithm based on Gaussian elimination, (Richtmyer [11]).

The high order correct method (H.O.C.M.) of Douglas [3] applied to (4) yields the scheme

$$
\begin{aligned}
(10 I+12 r A) \boldsymbol{\omega}_{i, n+1}+ & (I-6 r A)\left[\boldsymbol{\omega}_{i+1, n}+\boldsymbol{\omega}_{i-1, n+1}\right] \\
& =(10 I-12 r A) \boldsymbol{\omega}_{i, n}+(I+6 r A)\left[\boldsymbol{\omega}_{i+1, n}+\omega_{i-1, n}\right]
\end{aligned}
$$

which also requires the solution of a block tridiagonal system of equations of the form (13) at each time step. In this case, the matrices $A_{i}, B_{i},(i=1,2)$ are given by

$A_{1}=10 I+12 r A, \quad A_{2}=I-6 r A, \quad B_{1}=10 I-12 r A, \quad B_{2}=I+6 r A$.

It is easily shown that the local truncation errors of (12) and (15) are $O\left(h^{2}+k^{2}\right)$ and $O\left(h^{4}+k^{2}\right)$ respectively. The stability of these methods may be analysed by the method of Richtmyer which yields amplification matrices of the form

where

$$
G=\left[\begin{array}{cc}
\frac{1-d^{2}}{1+d^{2}} & \frac{2 d}{1+d^{2}} \\
\frac{-2 d}{1+d^{2}} & \frac{1-d^{2}}{1+d^{2}}
\end{array}\right]
$$

$$
d=2 r \sin ^{2}(\beta h / 2)
$$

for (12) and

$$
d=\frac{6 r \sin ^{2}(\beta h / 2)}{3-\sin ^{2}(\beta h / 2)}
$$

for (15). Since $G$ is a unitary matrix, it follows that both methods are unconditionally stable.

4. Calculation of the Solution of (1). Eq. (1) arises in the study of the transverse vibrations of a uniform flexible beam hinged at both ends. The quantities $\phi_{i, n}$ and $\psi_{i, n}$ are thus the values of the velocity and the bending moment respectively at the 
point $x=i h(i=1, \cdots, N-1)$ at time $t=n k(n=1,2, \cdots)$ and may, in fact, be the physical quantities we seek from the differential equation (1). If we also require the displacement $u_{i, n}$ at the point $(i h, n k$ ), this may be obtained from the values of $\phi_{i, n}$ and $\psi_{i, n}$ by means of the algorithm

$$
u_{i+1, n}-2 u_{i, n}+u_{i-1, n}=\left(h^{2} / 12\right)\left(\psi_{i+1, n}+10 \psi_{i, n}+\psi_{i-1, n}\right)
$$

which is based on the method of Numerov [9] for the solution of the second order ordinary differential equation

$$
y^{\prime \prime}=f(x, y) \text {. }
$$

If $\psi$ is calculated by the semiexplicit method (10) the local truncation error is $O\left(h^{5}\right)$; otherwise it is $O\left(h^{6}\right)$. A more accurate algorithm for use in conjunction with the high accuracy method (15) is

$$
\begin{aligned}
u_{i+1, n}-2 u_{i, n}+ & u_{i-1, n} \\
& =\frac{h^{2}}{12}\left(\psi_{i+1, n}+10 \psi_{i, n}+\psi_{i-1, n}\right)-\frac{h^{2}}{240 r} \delta_{x}{ }^{2}\left(\phi_{i, n}-\phi_{i, n-1}\right)
\end{aligned}
$$

which has a local truncation error of $O\left(h^{8}\right)$. In order to determine $u_{i, n}(i=1, \cdots$, $N-1$ ) from formula (16) or (17) a tridiagonal system of equations must be solved.

5. Variable Coefficient Case. Let us now consider the partial differential equation

$$
\frac{\partial^{2} u}{\partial t^{2}}+\frac{\partial^{2}}{\partial x^{2}}\left(\sigma(x) \frac{\partial^{2} u}{\partial x^{2}}\right)=0, \quad \sigma(x)>0,
$$

which, subject to the initial and boundary conditions (1b) and (1c), describes the transverse vibrations of a beam of variable density and/or cross-section. By the introduction of the new variables

$$
\Phi=\frac{\partial u}{\partial t}, \quad \Psi=\sigma(x) \frac{\partial^{2} u}{\partial x^{2}}
$$

Eq. (18) may be written as the second-order system

$$
\frac{\partial \Omega}{\partial t}=A(x) \frac{\partial^{2} \Omega}{\partial x^{2}}
$$

where

$$
\mathbf{\Omega}=\left[\begin{array}{l}
\Phi \\
\Psi
\end{array}\right] \text { and } A(x)=\left[\begin{array}{cc}
\cdot & -1 \\
\sigma(x) & \cdot
\end{array}\right] .
$$

The finite difference schemes described in Sections 1 and 2 of this paper can be easily generalized to this case of variable coefficients without loss of accuracy. Care must, however, be taken when generalizing the high accuracy scheme (15). Writing Eq. (19) in the form

$$
[A(x)]^{-1} \frac{\partial \Omega}{\partial t}=\frac{\partial^{2} \Omega}{\partial x^{2}}
$$


and following Douglas [12] we obtain the scheme

$$
\begin{aligned}
& \frac{1}{12}\left[A\left(x_{i+1}\right)\right]^{-1}\left(\omega_{i+1, n+1}-\omega_{i+1, n}\right)+\frac{5}{6}\left[A\left(x_{i}\right)\right]^{-1}\left(\omega_{i, n+1}-\omega_{i, n}\right) \\
& +\frac{1}{12}\left[A\left(x_{i-1}\right)\right]^{-1}\left(\omega_{i-1, n+1}-\omega_{i-1, n}\right)=\frac{1}{2} r \delta_{x}{ }^{2}\left(\omega_{i, n+1}+\omega_{i, n}\right)
\end{aligned}
$$

which is again correct to $O\left(h^{4}+k^{2}\right)$ and requires the solution of a block tridiagonal system of equations at each time step.

Since the differential equation (19) and hence the approximating difference equations have variable coefficients, no longer can we use the method of Richtmyer to examine stability. Using energy methods Lees [13] has established that the Crank-Nicolson method and high accuracy method of Douglas for the solution of (5) are unconditionally stable when generalised to the equation

$$
\frac{\partial v}{\partial t}=\sigma(x, t) \frac{\partial^{2} u}{\partial x^{2}} \text {. }
$$

By an extension of Lees' techniques it may be possible to discuss the stability of the generalisations to (19) of the methods outlined in Sections 2 and 3 of the present paper. This will, however, require further investigation.

6. Two-Space Variable Case. The partial differential equation

$$
\frac{\partial^{2} u}{\partial t^{2}}+\nabla^{4} u=0, \text { where } \nabla^{2} \equiv \frac{\partial^{2}}{\partial x^{2}}+\frac{\partial^{2}}{\partial y^{2}},
$$

subject to appropriate initial and boundary conditions arises in the study of transverse vibrations of a uniform plate and may be written in the form

$$
\frac{\partial \boldsymbol{\Omega}}{\partial t}=A \nabla^{2} \boldsymbol{\Omega}
$$

by introducing the variables

$$
\Phi=\frac{\partial u}{\partial t} \text { and } \Psi=\nabla^{2} u
$$

where

$$
\mathbf{\Omega}=\left[\begin{array}{l}
\Phi \\
\Psi
\end{array}\right] \text { and } A=\left[\begin{array}{cc}
\cdot & -1 \\
1 & \cdot
\end{array}\right]
$$

as before.

Eq. (22) can be solved by applying any of the finite difference techniques for the numerical solution of the corresponding scalar equation

$$
\frac{\partial v}{\partial t}=\nabla^{2} v
$$

For example, the alternating direction implicit (ADI) method of Peaceman and Rachford [10] when applied to Eq. (22) yields the algorithm

$$
\begin{aligned}
& {\left[I-\frac{1}{2} r A \delta_{x}{ }^{2}\right] \omega_{i, j, n+1}^{*}=\left[I+\frac{1}{2} r A \delta_{y}{ }^{2}\right] \omega_{i, j, n},} \\
& {\left[I-\frac{1}{2} r A \delta_{y}{ }^{2}\right] \omega_{i, j, n+1}=\left[I+\frac{1}{2} r A \delta_{x}{ }^{2}\right] \omega_{i, j, n+1}^{*}}
\end{aligned}
$$


in the usual notation where the local truncation errors are $O\left(h^{2}+k^{2}\right)$. The displacement $u_{i, j, n}$ at the poine $(i h, j h, n k)(i, j=1, \cdots, N-1 ; n=1,2, \cdots)$ may then be determined by solving at each time step the Poisson equation

$$
\nabla^{2} u=\Psi(x, y, t),
$$

by the Peaceman-Rachford iterative method

$$
\begin{aligned}
& {\left[1-\frac{1}{2} r \delta_{x}{ }^{2}\right] u_{i, j, n}^{(m+1)^{*}}=\left[1+\frac{1}{2} r \delta_{y}{ }^{2}\right] u_{i, j, n}^{m}-\frac{1}{2} r h^{2} \psi_{i, j, n}} \\
& {\left[1-\frac{1}{2} r \delta_{y}{ }^{2}\right] u_{i, j, n}^{(m+1) *}=\left[1+\frac{1}{2} r \delta_{x}{ }^{2}\right] u_{i, j, n}^{(m+1)}-\frac{1}{2} r h^{2} \psi_{i, j, n},}
\end{aligned}
$$

where $r$, previously equal to $k / h^{2}$, is now an iteration parameter.

A more accurate method of solving (22) is that of Mitchell and Fairweather [8] which, in its simplest form, that is, for the solution of (22) with zero boundary conditions, may be written as

$$
\begin{aligned}
& {\left[I-\frac{1}{2}\left(r A-\frac{1}{6} I\right) \delta_{x}{ }^{2}\right] \omega_{i, j, n+1}^{*}=\left[I+\frac{1}{2}\left(r A+\frac{1}{6} I\right) \delta_{y}{ }^{2}\right] \omega_{i, j, n}} \\
& {\left[I-\frac{1}{2}\left(r A-\frac{1}{6} I\right) \delta_{y}{ }^{2}\right] \omega_{i, j, n+1}=\left[I+\frac{1}{2}\left(r A+\frac{1}{6} I\right) \delta_{x}{ }^{2}\right] \omega_{i, j, n+1}^{*} .}
\end{aligned}
$$

This method has local truncation errors of $O\left(h^{4}+k^{2}\right)$.

Eq. (24) can then be solved by the iterative method based on (26) which takes the form

$$
\begin{aligned}
& {\left[1-\frac{1}{2}\left(r-\frac{1}{6}\right) \delta_{x}{ }^{2}\right] u_{i, j, n}^{(m+1)^{*}}=\left[1+\frac{1}{2}\left(r+\frac{1}{6}\right) \delta_{y}{ }^{2}\right] u_{i, j, n}^{m}-\frac{1}{2}\left(r-\frac{1}{6}\right) \bar{\psi}_{i, j, n}} \\
& {\left[1-\frac{1}{2}\left(r-\frac{1}{6}\right) \delta_{y}{ }^{2}\right] u_{i, j, n}^{(m+1)}=\left[1+\frac{1}{2}\left(r+\frac{1}{6}\right) \delta_{x}{ }^{2}\right] u_{i, j, n}^{(m+1)^{*}}-\frac{1}{2}\left(r+\frac{1}{6}\right) \bar{\psi}_{i, j, n}}
\end{aligned}
$$

where $\bar{\psi}_{i, j, n}=h^{2}\left[1+\frac{1}{12}\left(\delta_{x}^{2}+\delta_{y}{ }^{2}\right)\right] \psi_{i, j, n}$.

The stability of the ADI methods (23) and (26) may be examined by eliminating the auxiliary vector $\omega_{i, m, n+1}^{*}$ and applying the method of Richtmyer. For example, if $\omega_{i, j, n+1}^{*}$ is eliminated from (23), we obtain the equation

$$
\left[I-\frac{1}{2} r A \delta_{x}^{2}\right]\left[I-\frac{1}{2} r A \delta_{y}^{2}\right] \omega_{i, j, n+1}=\left[I+\frac{1}{2} r A \delta_{x}^{2}\right]\left[I+\frac{1}{2} r A \delta_{y}{ }^{2}\right] \omega_{i, j, n}
$$

which may be simplified to take the form

$$
\begin{aligned}
& {\left[\left(1-\frac{1}{4} r^{2} \delta_{x}{ }^{2} \delta_{y}{ }^{2}\right) I-\frac{1}{2} r A\left(\delta_{x}{ }^{2}+\delta_{y}{ }^{2}\right)\right] \omega_{i, j, n+1}} \\
& =\left[\left(1-\frac{1}{4} r^{2}{\delta_{x}}^{2} \delta_{y}{ }^{2}\right) I+\frac{1}{2} r A\left(\delta_{x}{ }^{2}+\delta_{y}{ }^{2}\right)\right] \omega_{i, j, n}
\end{aligned}
$$

since $A^{2}=-I$. If we now substitute

$$
\omega_{m, p, n}=\varrho_{m} e^{i \beta x} e^{i \gamma y}
$$

where $\varrho_{n}=\left[\begin{array}{l}\tilde{a}_{n} \\ \tilde{b}_{n}\end{array}\right]$ and $x=m h, y=p h(m, p=1, \cdots, N-1)$, simple manipulation yields

$$
\varrho_{n+1}=G \varrho_{n}
$$

where $G$ is again of the form of the unitary matrix of Section 3 with

$$
d=\frac{2 r\left[\sin ^{2}(\beta h / 2)+\sin ^{2}(\gamma h / 2)\right]}{1-4 r \sin ^{2}(\beta h / 2) \sin ^{2}(\gamma h / 2)},
$$

and hence the ADI method is unconditionally stable. A similar analysis applied to (26) yields a unitary amplification matrix of the same form as before with $d$ in 
this case given by

$$
d=\frac{2 r\left\{3\left[\sin ^{2}(\beta h / 2)+\sin ^{2}(\gamma h / 2)\right]-2 \sin ^{2}(\beta h / 2) \sin ^{2}(\gamma h / 2)\right\}}{3-\left[\sin ^{2}(\beta h / 2)\right]+\sin ^{2}(\gamma h / 2)-12\left(r^{2}-\frac{1}{36}\right) \sin ^{2}(\beta h / 2) \sin ^{2}(\gamma h / 2)},
$$

and thus the ADI method (26) is also unconditionally stable.

The convergence of the iterative procedure (25) has been discussed by several authors, for example, Birkhoff et al., [1], Varga [14], who have shown that the procedure converges for all positive values of the iteration parameter $r$. In fact, this parameter may be varied from iteration to iteration to accelerate convergence, (Birkhoff et al., [1]). Similar results have been established by Mitchell and Fairweather [8], [15] for the ADI method (27).

7. Numerical Experiments. The finite difference methods outlined in Sections 2 and 3 of the present paper are now used to solve a vibrating beam problem consisting of Eq. (3) together with the initial conditions

$$
u(x, 0)=\frac{x}{12}\left(2 x^{2}-x^{3}-1\right)
$$

$$
\frac{\partial}{\partial t} u(x, 0)=0 \text {, }
$$

$$
0 \leqq x \leqq 1,
$$

and the boundary conditions

$$
\begin{gathered}
u(0, t)=u(1, t)=0, \\
\frac{\partial^{2}}{\partial x^{2}} u(0, t)=\frac{\partial^{2}}{\partial x^{2}} u(1, t)=0,
\end{gathered}
$$

This is the problem considered by Evans, the theoretical solution being

$$
u(x, t)=\sum_{s=1}^{\infty} d_{s} \sin (2 s+1) \pi x \cos (2 s+1)^{2} \pi^{2} t
$$

where $d_{s}=-8 /(2 s+1)^{5} \pi^{5}$.

If we consider Eq. (4), the initial and boundary conditions may be derived from $(28 \mathrm{a})$ and $(28 \mathrm{~b})$ and are given by

$$
\boldsymbol{\Omega}(x, 0)=\left[\begin{array}{c}
0 \\
x-x^{2}
\end{array}\right], \quad 0 \leqq x \leqq 1,
$$

and

$$
\boldsymbol{\Omega}(0, t)=\boldsymbol{\Omega}(1, t)=\left[\begin{array}{l}
0 \\
0
\end{array}\right], \quad t \geqq 0,
$$

respectively.

In order to provide a comparison with Evans, we choose $h=0.05, k=0.00125$ and hence $r=\frac{1}{2}$. In Table I, the differences between the theoretical solution for $u$ given by (29) and the computed solution obtained by the stated methods are shown for $t=0.02, x=j h, j=1,2, \cdots, 10$. Similar results for the bending mornent $\Psi=\partial^{2} y / \partial x^{2}$ are quoted in Table II.

Since each method is unconditionally stable for all values of the mesh ratio $r$, 


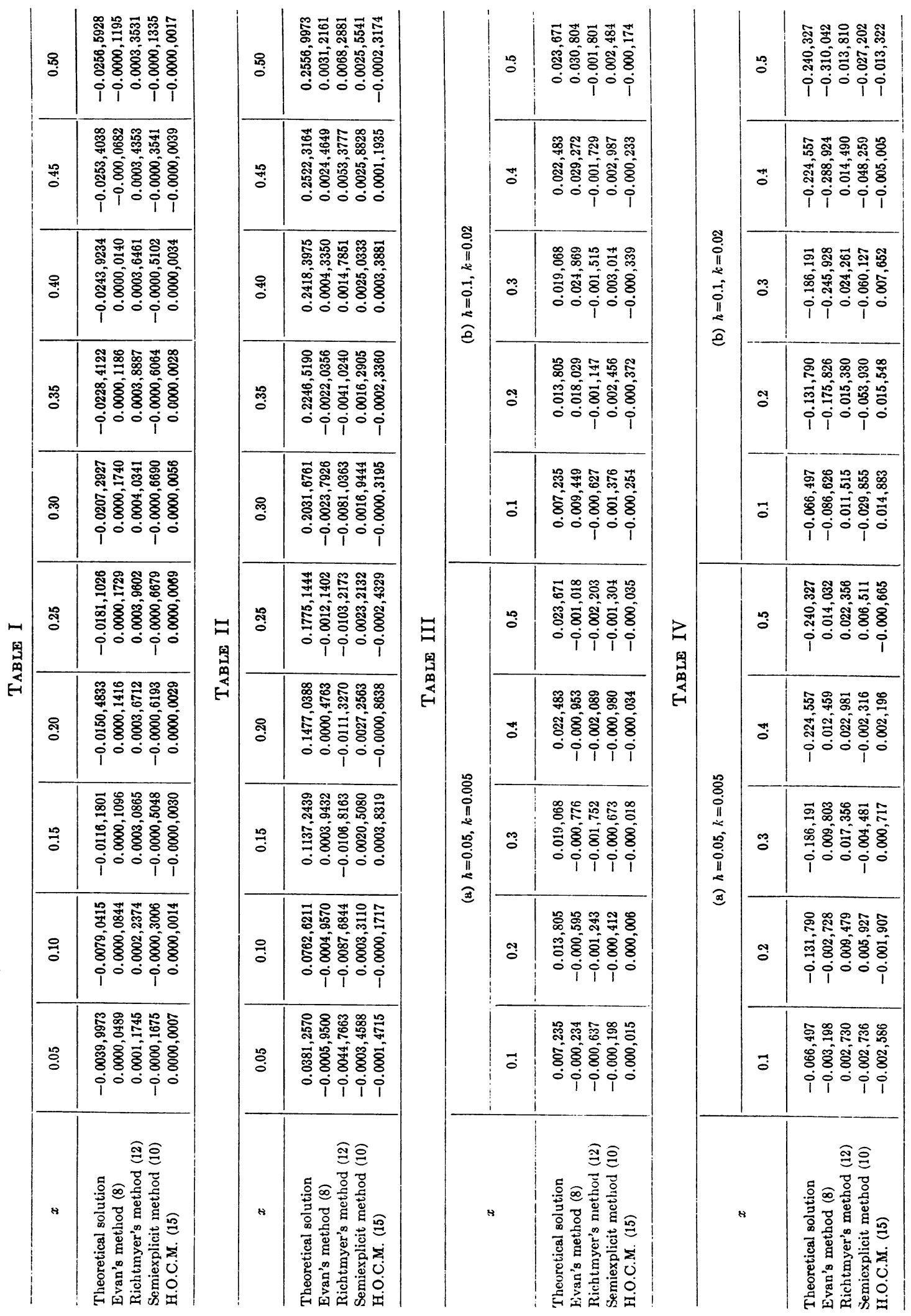


the choice $r=\frac{1}{2}$ is rather restrictive. Consequently in the second series of experiments, calculations are carried out to $t=1$ with $r=2$ and (a) $h=0.05, k=0.005$, and (b) $h=0.1, k=0.02$. The errors in the displacement $u$ and the bending moment $\Psi$ at the nodes $t=1, x=(0.1) j, j=1,2, \cdots, 5$ are quoted in Table III and Table IV respectively. It appears that for case (b) Evans' method is by far the least accurate due to the dominance in the local truncation error of $\Psi$ of the term $(k / h)^{2} \partial^{2} \Phi / \partial t^{2}$ which, for this problem, is equal to

$$
\left(\frac{k}{h}\right)^{2} \sum_{s=0}^{\infty} 8 \pi(2 s+1) \sin (2 s+1) \pi x \sin (2 s+1)^{2} \pi^{2} t .
$$

In case (b), $(k / h)^{2}=1 / 25$ while in case (a) and the first set of experiments $(k / h)^{2}$ has the values $1 / 100$ and $1 / 1600$ respectively.

Although the high order correct method of Douglas given by (15) is the most accurate, it requires the most computing time. The semi-explicit method (10) is seen to be as accurate as Evans' method when $(k / h)^{2}$ is small, and more accurate when $(k / h)^{2}$ is large. It requires about as much computing time as Evans' method and has the advantage of requiring starting data only on the initial line so that no extrapolation is necessary to find starting data on the line $t=k$. Richtmyer's method compares favorably with the explicit methods, but requires more computing time.

All calculations were carried out to ten places of decimals on the IBM 1620 computer of the University of St. Andrews.

Acknowledgment. Mr. A. R. Gourlay's share of the work was carried out whilst he was in receipt of a Carnegie Scholarship.

Department of Mathematics

Rice University

Houston, Texas 77001

Department of Applied Mathematics

University of St. Andrews

Fife, Scotland

1. G. Birkhoff, R. S. Varga \& D. M. Young, "Alternating direction implicit methods," Advances in Computers, Vol. 3, Academic Press, New York, 1962.

2. J. Crank \& P. Nicolson, "A practical method for numerical evaluation of solutions of partial differential equations of the heat-conduction type," Proc. Cambridge Philos. Soc., v. 43, 1947, pp. 50-67. MR 8, 409.

3. J. Dodglas, JR., "The solution of the diffusion equation by a high order correct difference equation,"J. Math. Phys., v. 35, 1956, pp. 145-151. MR 19, 884.

4. E. C. DU ForT \& S. P. Frankel, "Stability conditions in the numerical treatment of parabolic differential equations," $M T A C$, v. 7, 1953, pp. 135-152. MR 15, 474.

5. D. J. Evans, "A stable explicit method for the finite-difference solution of a fourthorder parabolic partial differential equation," Comput. J., v. 8, 1965, pp. 280-287.

6. H. O. KREISs, "Ubber implizite Differenzmethoden für partielle Differentialgleichungen," Numer. Math., v. 5, 1963, pp. 24-47. MR 27 *5376.

7. M. LEEs, "Alternating direction and semi-explicit difference methods for parabolic partial differential equations," Numer. Math., v. 3, 1961, pp. 398-412. MR 25 \#605.

8. A. R. Mitchell \& G. FAIRWEather, "Improved forms of the alternating direction methods of Douglas, Peaceman and Rachford for solving parabolis and elliptic equations," Numer. Math., v. 6, 1964, pp. 285-292. MR 30 * 4391.

9. B. Numerov, Publ. Observ. Astrophys. Central Russia, v. 2, 1933, p. 188.

10. D. W. PEACEMAN \& H. H. RACHForD, "The numerical solution of parabolic and elliptic differential equations", J. Soc. Indust. Appl. Math., v. 3, 1955, pp. 28-41. MR 17, 196.

11. R. D. RICHTMYER, "Difference methods for initial value problems," Interscience Tracts in Pure and Applied Mathematics, Tract 4, Interscience, New York, 1957. MR 20 * 438. 
12. J. Douglas, JR., "The application of stability analysis in the numerical solution of quasi-linear parabolic differential equations," Trans. Amer. Math. Soc., v. 89, 1958, pp. 484518. MR 24 *A1521.

13. M. LEES, "A priori estimates for the solutions of difference approximations to parabolic partial differential equations," Duke Math. J., v. 27, 1960, pp. 297-312. MR 22 *12725.

14. R. S. VArgA, Matrix Iterative Analysis, Prentice-Hall, Englewood Cliffs, N. J., 1962. MR 28 *1725.

15. G. Fairweather \& A. R. Mitchell, "Some computational results of an improved ADI method for the Dirichlet problem," Comput. J. (To appear.) 